\title{
Gestión del tiempo en alumnado universitario con diferentes niveles de rendimiento académico
}

Angélica Garzón Umerenkova ${ }^{1}$

Javier Gil Flores ${ }^{2}$

\section{Resumen}

En este trabajo se estudia la relación entre gestión del tiempo y rendimiento académico en el alumnado universitario. La hipótesis de partida es que los estudiantes con alto nivel de habilidades en gestión del tiempo obtienen mejores resultados de aprendizaje. Se ha utilizado una muestra de 494 estudiantes universitarios de primer año, en dos universidades colombianas. A esta muestra se administró una adaptación al español del Time Management Behavior Questionnaire (TMBQ), y se recogieron datos sobre las calificaciones académicas en su primer año en la universidad. De manera complementaria, se han celebrado cuatro grupos focales con alumnado de bajo y alto nivel de rendimiento, accediendo a sus experiencias, opiniones y valoraciones en relación con la gestión del tiempo. Los resultados obtenidos indican la existencia de una cierta relación positiva entre las dos variables analizadas, en línea con los hallazgos de estudios previos. Al comparar la gestión del tiempo entre subgrupos de estudiantes que alcanzan las mejores y peores calificaciones, se observan diferencias a favor de los primeros, particularmente en la sub-escala relativa a sus percepciones sobre el control del tiempo. Los discursos de grupo reflejan también mejores conductas autorregulatorias en el manejo del tiempo por parte del alumnado de alto nivel de rendimiento. De acuerdo con estos resultados, se hacen recomendaciones sobre el entrenamiento del alumnado universitario de primer año en estrategias para la gestión del tiempo.

\section{Palabras clave}

Gestión del tiempo - Rendimiento académico - Educación superior - Métodos cuantitativos y cualitativos.

\section{Time management in university students with different levels of academic performance}

\section{Abstract}

In this work, we have analyzed the relationship between time management and academic performance in university students. Our initial hypothesis is that students with high level of

\footnotetext{
1- Universidad El Bosque, Bogotá, Colombia. Contacto: garzonangelica@unbosque.edu.co

2- Universidad de Sevilla, Sevilla, España. Contacto: jflores@us.es

DOl: http://dx.doi.org/10.1590/S1678-4634201708157900
} 
skills in time management achieve better learning outcomes. The sample consisted of 496 freshman students from two Colombian Universities. We have used a Spanish version of the Time Management Behavior Questionnaire (TMBQ) and we have taken the students' average academic grades from their first year in the university. To complement this, four focus groups were carried out with students with low or high performance levels, allowing us to know their perspectives about time management. Results show a weak relationship between both variables utilized, in accordance with the results of previous studies. Students with higher grades present better time management than students with low grades, particularly in the sub-scale about perceptions of time control. Group discourses allow us to identify better auto-regulatory time management behaviors in high level performance students. Consequently, we recommend training freshman undergraduates in time management strategies.

\section{Keywords}

Time management - Academic performance - Higher Education - Quantitative and qualitative methods

\section{Introducción}

El rendimiento académico de los estudiantes constituye un elemento central en la enseñanza universitaria, dado que representa una medida del aprendizaje logrado por los individuos y, al mismo tiempo, un indicador de calidad de las instituciones y sistemas de educación superior. De ahí que su estudio constituya un objeto de especial interés para la investigación en el ámbito de la educación superior. Entre las variables demográficas, personales, académicas, institucionales o contextuales que podrían vincularse a los resultados de aprendizaje, la gestión del tiempo por los estudiantes ha recibido atención creciente en las últimas décadas, como así lo indica la revisión de trabajos realizada por Claessens y otros autores (2007).

La gestión del tiempo académico suele considerase un componente importante de la autorregulación del aprendizaje (EILAM; AHARON, 2003; ZIMMERMAN, 1996), que hace referencia al modo en que el alumnado regula su tiempo de estudio para alcanzar exitosamente los objetivos educativos propuestos (PINTRICH et al., 1993). Como señalan Liu y otros autores (2009), la gestión del tiempo se ha definido y operativizado para fines investigativos de muy variadas formas, aunque convergentes en una idea central: la finalización de tareas o actividades en un tiempo esperado, obteniendo resultados de calidad, mediante procedimientos tales como la planificación, la organización o la priorización. Tras la revisión de los estudios empíricos sobre manejo del tiempo publicados desde 1982 hasta 2004, Claessens y otros autores (2007) concluyen que la gestión del tiempo se refiere a conductas que buscan lograr un efectivo uso del tiempo, mientras se ejecutan actividades dirigidas a una meta. Dentro de este concepto se incluye una serie de actuaciones de planificación, seguimiento y regulación eficaz del tiempo, haciendo uso para ello de estrategias como el manejo de una agenda, la planificación diaria o la priorización de 
diferentes actividades. La gestión del tiempo académico supone el establecimiento y logro de metas, al tiempo que implica una supervisión y regulación de las metas establecidas.

Contemplada desde los modelos de autorregulación académica, la gestión del tiempo tiene un fuerte componente motivacional, de autocontrol, y también metacognitivo. El estudiante debe valorar y tomar decisiones sobre aspectos tales como el orden en que va a abordar las diferentes tareas y el tiempo que va a dedicar a cada tema de estudio. Generalmente, los alumnos tienden a emplear más tiempo en los temas en que se encuentran a mayor distancia del estado de aprendizaje deseado (DUNLOSKY; HERTZOG, 2000; SON; KORNELL, 2009). No obstante, cuando actúan bajo presión por falta de tiempo, la estrategia más utilizada por los estudiantes, y la más efectiva, podría ser distribuir el tiempo entre los tópicos relativamente fáciles que aún no se hayan aprendido o estudiado (METCALFE; KORNELL, 2005).

Las habilidades de gestión del tiempo pueden variar entre muestras específicas de estudiantes. Se han encontrado mayores niveles de habilidad en las alumnas que en los alumnos (DURÁN-APONTE; PUJOL, 2013; KAYA et al., 2012; PEHLIVAN, 2013; SOARES; ALMEIDA; GUISANDEZ, 2011), entre estudiantes de mayor edad, o entre quienes trabajan (FORBUS; NEWBOLD; MEHTA, 2011; MACAN et al., 1990; ROBOTHAM, 2012). Se han encontrado evidencias que relacionan positivamente la gestión del tiempo con la memoria, la creatividad, el sentido de coherencia, el autocontrol o la autoestima, entre otros rasgos personales (LIU et al., 2009; MACAN; GOBSON; CUNNINGHAM, 2010).

El tiempo disponible para el estudiante universitario es un recurso limitado, cuya adecuada utilización conlleva abarcar las diferentes tareas académicas y hacerlo dentro de los plazos establecidos. Cabe suponer que una adecuada gestión de este recurso podría conducir a lograr el éxito en los estudios. En el presente trabajo, nuestro interés se centra en la relación entre gestión del tiempo y rendimiento. La investigación empírica desarrollada hasta ahora sobre este tema, ha considerado tanto la cantidad de tiempo empleado como el modo en que éste se gestiona, valorando su incidencia en el rendimiento. Ukpong y George (2013) encontraron importantes diferencias entre los resultados académicos de los estudiantes universitarios según el tiempo dedicado al estudio. Aunque este resulte un factor a tener en cuenta, la clave de un buen rendimiento no está en estudiar cuantas más horas mejor. La manera en que los estudiantes gestionan su tiempo de estudio es tan importante, o incluso más, que el tiempo que emplean en el mismo (WILHITE, 1990). De hecho, la gestión del tiempo constituye uno de los predictores del rendimiento académico más relevantes (GEORGE et al., 2008).

Entre los trabajos que se centran sobre la relación entre gestión del tiempo y rendimiento más citados en la literatura, se encuentran los realizados a principios de los noventa (BRITTON; TESSER, 1991; MACAN et al., 1990). Macan y otros autores (1990) construyeron y administraron el Time Management Behavior Questionnaire (TMBQ), recogiendo además las calificaciones académicas de los estudiantes a partir de la información al respecto aportada por ellos mismos. La correlación positiva entre las puntuaciones en el TMBQ y las calificaciones resultó significativa. En el estudio de Britton y Tesser (1991) se obtuvieron similares resultados, administrando un cuestionario sobre gestión del tiempo y recogiendo una medida más robusta del rendimiento. En ese trabajo, el rendimiento fue calculado como media de las calificaciones obtenidas por los estudiantes durante cuatro cursos académicos. Investigaciones más recientes 
han arrojado resultados que apuntan en la misma dirección (BASILA, 2014; DURÁN-APONTE; PUJOL, 2012; GARCÍA-ROS; PÉREZ-GONZÁLEZ, 2012; MACCANN; FOGARTY; ROBERTS, 2012), subrayando la vinculación entre resultados académicos y manejo del tiempo. Por otra parte, se ha apreciado un incremento del rendimiento del alumnado tras participar en programas focalizados en la mejora de sus competencias académicas y en la gestión del tiempo (KITSANTAS; WINSLER; HUIE, 2008; PEHLIVAN, 2013; ROBBINS et al., 2009). Sin embargo, no todas las evidencias acumuladas ratifican esta relación; en algunos estudios no se han hallado relaciones significativas entre habilidades de gestión del tiempo y rendimiento académico (HAMDAN et al., 2014; OLOWOOKERE et al., 2015). En otros, a pesar de constatarse la vinculación entre ambas variables, esta resultó ser de una intensidad baja (BARRERA; DONOLO; RINAUDO, 2008; CLAESSENS et al., 2007).

En su revisión de investigaciones sobre la gestión del tiempo en los ámbitos académico y laboral, Claessens y otros autores (2007) señalaban la necesidad de incrementar la investigación sobre este tópico. Respondiendo a esa directriz, y saliendo al paso de las evidencias de distinto signo halladas hasta el momento, el presente trabajo aborda el estudio de las conductas de gestión del tiempo exhibidas por el alumnado universitario. Nos centramos en su relación con el rendimiento, tratando de profundizar en la comprensión del modo en que estructuran el tiempo los estudiantes caracterizados por diferentes niveles de rendimiento académico. Concretamente, nos proponemos responder a los siguientes interrogantes: ¿existe relación entre la gestión del tiempo académico y el rendimiento logrado por los estudiantes universitarios?, ¿existen diferencias entre alumnado de bajo y alto rendimiento académico en lo que respecta a la gestión del tiempo?, ¿qué conductas y opiniones caracterizan al alumnado de niveles bajo y alto de rendimiento en cuanto a la gestión que hacen de su tiempo académico?

\section{Método}

\section{Diseño}

En la citada revisión de Claessens y otros autores (2007) se constata la utilización mayoritaria de cuestionarios, y en menor medida experimentos, como principales métodos o técnicas de investigación. En el caso de los estudios sobre la gestión del tiempo en el ámbito universitario, únicamente en tres trabajos se adoptaron metodologías cualitativas, recurriendo en todos ellos a la elaboración de diarios. Los citados autores señalaban la necesidad de realizar estudios de corte cualitativo, que aporten información complementaria sobre las conductas de gestión del tiempo aplicadas por los sujetos.

Asumiendo esta propuesta, en el presente trabajo se integran las metodologías cuantitativa y cualitativa para acercarnos al estudio de la relación entre gestión del tiempo y rendimiento. Desde el punto de vista cuantitativo, se adopta un diseño ex-post facto correlacional, basado en la aplicación de un cuestionario y en la recogida de registros oficiales sobre rendimiento. De manera complementaria, se desarrolla un estudio cualitativo apoyado en la metodología 
de investigación mediante grupos focales, mediante el cual se accede a las perspectivas del alumnado sobre el manejo del tiempo en sus estudios universitarios.

\section{Participantes}

Han participado en el estudio cuantitativo 494 estudiantes de primer año (367 alumnos y 127 alumnas), de las universidades El Bosque y Uniempresarial, ambas radicadas en la ciudad de Bogotá. Las edades de los sujetos participantes se encuentran comprendidas entre los 16 y 46 años (media=19,31; desviación típica=3,56). Se trata de una muestra no probabilística seleccionada en función de su accesibilidad, si bien aseguramos la heterogeneidad de la misma al incluir estudiantes de una diversidad de titulaciones: Psicología, Educación Bilingüe, Pedagogía, Ingeniería de Sistemas, Ingeniería Electrónica, Administración de Empresas, Finanzas y Comercio Exterior, Negocios Internacionales y Marketing. En el estudio cualitativo se incluyeron 25 estudiantes (17 alumnas y 8 alumnos), con edades comprendidas entre los 18 y 25 años, que se encontraban cursando diversas titulaciones universitarias.

Los estudiantes participaron de manera voluntaria en la investigación. Para ello, firmaron un documento con el que ratificaban haber recibido información sobre el propósito y la naturaleza del estudio, y expresaban estar de acuerdo en participar. Para quienes eran menores de edad, el consentimiento informado lo firmaron padres, madres o tutores legales.

\section{Instrumentos y técnicas}

La medida de la gestión del tiempo se apoya en el TMBQ diseñado por Macan y otros autores (1990). Consta de 34 ítems y utiliza una escala de respuesta tipo Likert de cinco puntos: nunca (1), pocas veces (2), algunas veces (3), habitualmente (4), siempre (5). De acuerdo con la estructura factorial del instrumento, se diferencian cuatro subescalas: establecimiento de objetivos y prioridades, herramientas para la gestión del tiempo, percepción sobre el control del tiempo, y preferencias por la desorganización. En su aplicación a estudiantes colombianos, se partió de la adaptación al español validada por García-Ros y Pérez-González (2012), quienes encontraron para las cuatro subescalas mencionadas indices de fiabilidad alfa de Cronbach de 0,90, 0,88, 0,70 y 0.68 respectivamente. En el presente estudio, se sustituyeron algunos términos y expresiones ajenos al contexto cultural colombiano, que fueron identificados en una aplicación piloto a cinco estudiantes. Aplicado el instrumento a la muestra estudiada, los índices de fiabilidad alfa alcanzaron los valores 0,82, 0,83, 0,71 y 0,67 en las cuatro sub-escalas, con niveles próximos a los obtenidos en estudios previos.

La otra variable implicada es el rendimiento. Hemos definido operativamente el rendimiento académico como promedio de las calificaciones obtenidas por los estudiantes al finalizar su primer semestre en la universidad. El valor máximo posible que podría llegar a alcanzar esta variable es 5. Los datos correspondientes fueron ofrecidos por las Secretarías Académicas de cada una de las Facultades en las que estudia el alumnado participante. 
Además de medir las variables relativas a gestión del tiempo y rendimiento, se han utilizado los grupos focales como técnica de investigación cualitativa. Se constituyeron cuatro grupos, de los cuales dos estaban integrados por estudiantes de alto rendimiento y dos por estudiantes de rendimiento bajo. En los grupos se procuró conseguir un cierto grado de heterogeneidad, garantizando la presencia de alumnos y alumnas de distintas titulaciones, tanto de jornada diurna como nocturna.

\section{Procedimiento}

Los estudiantes fueron reunidos en aulas de informática de sus respectivos centros, invitándolos a completar el TMBQ a través de computadora. Durante la aplicación, un investigador estuvo presente para resolver dudas que pudieran surgir a los encuestados.

La captación de participantes en los grupos focales, constitución de los grupos, convocatoria y desarrollo de las reuniones se realizaron de acuerdo con las pautas habitualmente indicadas para la implementación de esta técnica. Siguiendo las recomendaciones de Krueger y Casey (2014), las tareas llevadas a cabo son las que pasamos a describir:

a) Captación de participantes. El reclutamiento de los participantes se hizo acudiendo a clases de primer año de los distintos programas académicos. Tras informar sobre los objetivos y naturaleza del estudio, se elaboró un listado de individuos dispuestos a participar, consignando nombre, titulación y datos de contacto.

b) Constitución de los grupos y convocatoria. Posteriormente, fueron seleccionados estudiantes que encajaban en los perfiles requeridos para los grupos, y se estableció contacto telefónico con ellos convocándolos a las reuniones. Un criterio clave de inclusión en los grupos fue el nivel de rendimiento del estudiante, que se basó en el auto-reporte sobre el promedio (de 0 a 5) logrado en las asignaturas cursadas durante el primer semestre. Esta información se complementaba con el reporte de alguno de sus profesores. Para que un alumno fuera incluido en los grupos de alto rendimiento, debían darse dos circunstancias: que el profesor proporcionara un reporte positivo sobre el rendimiento académico en su asignatura y que el estudiante reportara un promedio superior a 4,2 en el primer semestre. En el momento de convocar a los participantes, se preguntó a los estudiantes de bajo rendimiento si durante el semestre habían sufrido problemas familiares o personales que afectaran a sus estudios. En función de esta información, podrían ser descartados los casos de bajo rendimiento motivados por factores circunstanciales.

c) Desarrollo de las reuniones. Las discusiones de grupo tuvieron lugar en la Facultad de Psicología, de la Universidad El Bosque, con duración de aproximadamente una hora. La sala utilizada disponía de una mesa redonda con capacidad para doce personas. Al inicio se recordó el propósito de la reunión, su carácter de discusión libre no dirigida, y se propuso como tema las actitudes, hábitos y estrategias del alumnado en relación con la gestión del tiempo. El moderador contó con una lista de temas auxiliares, recogiendo en parte los contenidos del TMBQ. Estos temas serían planteados en caso de no ser abordados durante la discusión. En un principio, el 
moderador de los grupos se limitó a plantear el tema, permitiendo una discusión libre entre los participantes. Solo intervino cuando la conversación se detenía o derivaba hacia áreas ajenas al tema. La sesión finalizaba al agotarse los temas auxiliares $\mathrm{y}$ cuando los participantes no planteaban nuevas experiencias, ideas $\mathrm{u}$ opiniones relativas a la gestión del tiempo. Los discursos generados fueron grabados en audio, con previo consentimiento de los participantes, y posteriormente transcritos para su análisis.

\section{Análisis de datos}

Partimos de una valoración inicial de la relación entre gestión del tiempo y rendimiento mediante el cálculo del coeficiente de correlación de Pearson. Para diferenciar alumnado de alto y bajo rendimiento, se realizó una partición de la muestra tomando como puntos de corte los cuartiles correspondientes a la distribución de esta variable. Calculamos estadísticos descriptivos (media y desviación estándar) para las puntuaciones en el TMBQ y en las cuatro sub-escalas, considerando tanto el conjunto de participantes como las dos sub-muestras generadas en función del nivel de rendimiento (bajo o alto). La comparación de medias obtenidas en los dos grupos de estudiantes de altas y bajas calificaciones se realizó mediante la prueba $\mathrm{T}$ para grupos independientes. El tamaño del efecto fue estimado mediante el estadístico d de Cohen. En estos análisis se utilizó el programa SPSS v.22, salvo para la obtención del estadístico d de Cohen, cuyo valor fue calculado mediante la fórmula propuesta por este autor (COHEN, 1988).

Para los discursos de grupo, se aplicaron técnicas de análisis de contenido. Partimos de una reducción de los datos mediante categorización temática, tomando como sistema inicial de categorias los temas auxiliares considerados al moderar las discusiones grupales. Estas categorías son las siguientes:

- Distribución del tiempo. Modo en que se estructuran las actividades académicas o extra académicas que el estudiante afronta cotidianamente.

- Planificación de tareas. Descripción de conductas o estrategias dirigidas al establecimiento de metas, la jerarquización de las tareas y el seguimiento de las mismas para lograr unos objetivos o metas definidos.

- Uso de herramientas. Empleo de instrumentos o técnicas para planificar las actividades en un periodo de tiempo determinado, contribuyendo a la gestión del tiempo.

- Valoraciones sobre la gestión del tiempo. Opiniones sobre el valor atribuido a la gestión del tiempo, así como valoraciones personales del estudiante acerca del grado de control que tiene sobre su propio tiempo académico.

La codificación se realizó por tanto de manera deductiva, asignando a una categoría los fragmentos de discurso con afinidad temática a la misma. Una vez codificados los discursos de grupo, examinamos los datos incluidos en cada categoría. Mediante la comparación interna de elementos se identificaron los significados relevantes, que fueron además diferenciados en función de su procedencia de grupos de alumnado con bajo o alto rendimiento. 


\section{Resultados}

\section{Gestión del tiempo y rendimiento}

De acuerdo con los resultados mostrados en la Tabla 1, la puntuación media en la escala TMBQ se sitúa en 3,34, algo por encima del valor 3 que representa el punto medio en la escala de respuesta utilizada. Atendiendo a las sub-escalas definidas para este instrumento, contrasta la baja puntuación registrada en el uso de herramientas para la gestión del tiempo (media=2,89) frente a las alcanzadas en las sub-escalas establecimiento de objetivos y prioridades (media $=3,46)$, percepción sobre el control del tiempo (media=3,53) $y$ preferencias por la desorganización (media=3,78). En esta última se incluían ítems de carga negativa, para los que fue invertida la escala de puntuación. En consecuencia, la elevada media registrada en preferencias por la desorganización indica, en realidad, una baja preferencia de los estudiantes por el desorden y la desorganización.

Tabla 1 - Estadísticos descriptivos para las puntuaciones en gestión del tiempo.

\begin{tabular}{|c|c|c|c|c|c|}
\hline & & & & Límite inferior & Límite superior \\
\hline Establecimiento de objetivos y prioridades & 471 & 3,46 & 0,63 & 3,40 & 3,52 \\
\hline Herramientas para la gestión del tiempo & 466 & 2,89 & 0,87 & 2,81 & 2,97 \\
\hline Percepción sobre el control del tiempo & 474 & 3,53 & 0,67 & 3,47 & 3,59 \\
\hline Preferencias por la desorganización & 476 & 3,78 & 0,86 & 3,70 & 3,86 \\
\hline Total de la escala TMBQ & 454 & 3,34 & 0,49 & 3,30 & 3,39 \\
\hline
\end{tabular}

$\mathrm{N}$ = número de estudiantes que responden a todos los ítems de la escala o sub-escalas

Fuente: Datos de la investigación

En cuanto a la variable rendimiento, el promedio de calificaciones obtenidas por los estudiantes en el primer semestre se sitúa en valores comprendidos entre un mínimo de 1,4 y un máximo de 4,7, alcanzándose una media de 3,71 (desviación estándar=0,46; intervalo de confianza al 95\%:3,67-3,75). La correlación de esta variable con la puntuación obtenida en el TMBQ alcanzó el valor $\mathrm{r}=0,122$, indicando una relación de baja intensidad, aunque estadísticamente significativa $(\mathrm{p}<0,01)$.

Tomando los cuartiles para la distribución de la variable rendimiento académico $(\mathrm{Q} 1=3,5, \mathrm{Q} 2=3,8$ y $\mathrm{Q} 3=4,0)$, en esta muestra se ha considerado que el rendimiento es relativamente bajo cuando el promedio del estudiante no alcanza el valor $3.5 \mathrm{y}$ alto cuando supera el valor 4.0. De este modo, quedan delimitadas dos sub-muestras con niveles de rendimiento claramente diferenciados, incluyendo respectivamente al $25 \%$ de estudiantes que obtuvieron las calificaciones más bajas y al 25\% que logra las mejores calificaciones. En la Tabla 2 se presentan estadísticos descriptivos para las puntuaciones en gestión del tiempo, correspondientes al alumnado de bajo y alto rendimiento identificados de este modo. Se incluyen también los resultados obtenidos al aplicar la prueba $\mathrm{T}$ para la comparación de medias y se calcula el tamaño del efecto mediante el estadístico $d$. 
Tabla 2 - Comparación de puntuaciones medias en gestión del tiempo entre grupos de bajo y alto rendimiento.

\begin{tabular}{|c|c|c|c|c|c|}
\hline & \multicolumn{2}{|c|}{ Media (Desviación estándar) } & \multirow[b]{2}{*}{$\mathrm{T}$} & \multirow[b]{2}{*}{$\mathrm{p}$} & \multirow[b]{2}{*}{$\begin{array}{l}\mathrm{d} \text { de } \\
\text { Cohen }\end{array}$} \\
\hline & $\begin{array}{c}\text { Nivel bajo } \\
(n=111) \\
(n=110)\end{array}$ & $\begin{array}{l}\text { Nivel alto } \\
(n=110)\end{array}$ & & & \\
\hline Establecimiento de objetivos y prioridades & $3,49(0,59)$ & $3,48(0,66)$ & 0,08 & 0,936 & 0,015 \\
\hline Herramientas para la gestión del tiempo & $2,80(0,87)$ & $3,01(0,86)$ & $-1,75$ & 0,081 & $-0,243$ \\
\hline Percepción sobre el control del tiempo & $3,37(0,72)$ & $3,65(0,68)$ & $-2,94$ & 0,004 & $-0,400$ \\
\hline Preferencias por la desorganización & $3,71(0,86)$ & $3,76(0,86)$ & $-0,45$ & 0,653 & $-0,058$ \\
\hline Total de la escala TMBQ & $3,30(0,43)$ & $3,41(0,53)$ & $-1,72$ & 0,087 & $-0,228$ \\
\hline
\end{tabular}

$\mathrm{T}$ = estadístico de contraste para la prueba t de Student

$\mathrm{p}=$ grado de significación

$\mathrm{d}$ de Cohen = medida del tamaño del efecto

Fuente: Datos de la investigación

En lo que respecta a la gestión del tiempo, la puntuación total en el TMBQ y las puntuaciones en tres de las sub-escalas señalan una superioridad del alumnado de alto rendimiento sobre el alumnado de bajo rendimiento. No obstante, solo llega a ser estadísticamente significativa $(\mathrm{p}<0,01)$ la diferencia observada en la sub-escala percepción sobre el control del tiempo $(\mathrm{T}=-2,941 ; \mathrm{p}<0,01 ; \mathrm{d}=-0,40)$. Los alumnos con altas calificaciones consideran, en mayor grado que los de bajas calificaciones, que ellos controlan y gestionan de una manera eficaz su tiempo. El tamaño del efecto para esta diferencia alcanza un nivel medio-bajo, de acuerdo con los criterios propuestos por Cohen (1988), para quien el valor $d=0,20$ implica un efecto pequeño y el valor $d=0,50$ representa un efecto medio o moderado.

\section{Perspectivas de los estudiantes sobre la gestión del tiempo}

La información aportada por los estudiantes en los grupos focales permite complementar el significado de las diferencias observadas en el análisis estadístico previo. Los principales resultados obtenidos en el análisis de los discursos de grupo han sido sintetizados en el cuadro 1, donde se incluyen algunas citas textuales representativas. El modo en que distribuyen su tiempo los estudiantes constituye un elemento principal en la diferenciación entre alumnado de bajo y alto rendimiento. Quienes alcanzan buenos resultados académicos pueden llegar a configurar patrones o hábitos que definen una estructura temporal semanal, diferenciando los momentos o días dedicados al estudio. Esta estructuración de horarios, separando trabajo académico y otras actividades, no está presente en el alumnado de bajo nivel de rendimiento, que acaba dejando sus tareas para el último momento.

En lo que respecta a la planificación de tareas, ambos tipos de alumnado evalúan los requerimientos temporales de las tareas académicas que han de afrontar a corto o medio plazo, ajustándolas temporalmente en función de su mayor o menor prioridad. Sin embargo, entre el alumnado de bajo rendimiento esta planificación puede no llegar a cumplirse en la práctica. Una consecuencia de la planificación fallida es la acumulación 
de tareas y actividades académicas conforme avanza el semestre, lo cual genera en el alumnado una percepción de falta de control sobre su tiempo y lleva a un bajo nivel de calidad en la ejecución, que deriva en pobres resultados académicos.

Cuadro 1 - Principales diferencias en la gestión del tiempo entre el alumnado de alto y bajo rendimiento.

\begin{tabular}{|c|c|c|}
\hline & Alumnado de alto rendimiento & Alumnado de bajo rendimiento \\
\hline \multirow{2}{*}{ 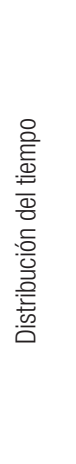 } & $\begin{array}{l}\text { "Voy adelantando cada día, o hasta los sábados[...] Después el resto } \\
\text { de fin de semana me desconecto totalmente" }\end{array}$ & $\begin{array}{l}\text { Abordan actividades de distinta naturaleza sin ajustarse a rutinas } \\
\text { temporales fijas. Tienden a procrastinar } \\
\text { "Lo primero que uno tiene que hacer es reconocer que uno no } \\
\text { tiene como un horario para realizar las actividades" } \\
\text { "Uno deja a última hora todo y ya queda apretado donde no } \\
\text { puede más" }\end{array}$ \\
\hline & $\begin{array}{l}\text { Separan el tiempo dedicado al estudio del tiempo de ocio, } \\
\text { concentrando éste al final de la semana } \\
\text { "Estudio [durante la semana] para dejar el domingo con tiempo libre" }\end{array}$ & $\begin{array}{l}\text { Realizan tanto actividades de ocio como de estudio a lo largo } \\
\text { de la semana } \\
\text { "Y te dicen vamos a comer perro y hablamos. Y cuando llegamos } \\
\quad \text { no hicimos la tarea, y uno no aprovecha el tiempo" }\end{array}$ \\
\hline 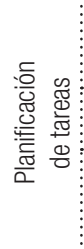 & $\begin{array}{l}\text { Realizan una previsión de tareas, las priorizan y las ubican en el } \\
\text { tiempo disponible } \\
\text { "Miro lo que me hace falta de trabajos y organizo para llegar al día" } \\
\text { "Manejo las cosas que tengo a largo plazo para ir haciendo a poquitos" }\end{array}$ & $\begin{array}{l}\text { Planifican las tareas de manera menos sistemática, o bien no } \\
\text { consiguen cumplir con la planificación } \\
\text { "No llevo una planificación escrita, [...] planifico como de un } \\
\text { día para otro" "Planifico qué debo hacer en la semana, en qué } \\
\text { debo invertir mi tiempo, pero se me dificulta mucho porque dejo } \\
\text { muchas cosas para último momento" }\end{array}$ \\
\hline \multirow{3}{*}{ 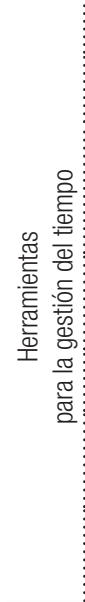 } & $\begin{array}{l}\text { Amplia variedad de herramientas, incluyendo recursos tecnológicos } \\
\text { "Hago como mapitas conceptuales, de lo que tengo pendiente" }\end{array}$ & $\begin{array}{l}\text { Menor variedad de herramientas, con predominio del uso de } \\
\text { herramientas tecnológicas frente a las basadas en el lápiz y } \\
\text { papel }\end{array}$ \\
\hline & $\begin{array}{l}\text { "Utilizo un cuaderno, lo tengo organizado por días [...] pongo, por } \\
\text { ejemplo, tengo pendiente tal trabajo y tengo que investigar tal tema" } \\
\text { "En la noche organizo con papeles de colores las materias, si tengo } \\
\text { que hacer un trabajo y para cuándo" } \\
\text { "Utilizo el celular, para tal día tal tarea, y lo que tengo que hacer" }\end{array}$ & $\begin{array}{l}\text { "Utilizo el iPod, [... ] y así controlo bien el tiempo } \\
\text { "En mi caso pongo alarmas en el celular" }\end{array}$ \\
\hline & $\begin{array}{l}\text { Utilización de herramientas para la planificación y para comprobar el } \\
\text { cumplimiento de tiempos y logro de metas } \\
\text { "Coloco todo lo que tengo para la semana, y voy tachando y si alguna } \\
\text { cosa me falta pongo un asterisco en rojo" }\end{array}$ & $\begin{array}{l}\text { Utilización de herramientas como sistemas de recuerdo, no } \\
\text { integradas en una planificación de tareas } \\
\text { "Utilizo el iPod, porque a mí se me olvidan mucho las cosas" }\end{array}$ \\
\hline 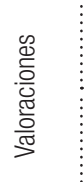 & $\begin{array}{l}\text { Atribuyen valor a la gestión del tiempo en la consecución del éxito } \\
\text { académico } \\
\text { "Pienso que la administración del tiempo es clave para hacer las } \\
\text { cosas bien" }\end{array}$ & $\begin{array}{l}\text { "Uno debe organizar el tiempo [...]. La mayoría de estudiantes } \\
\text { somos conscientes de que no administramos bien el tiempo" }\end{array}$ \\
\hline
\end{tabular}

Nota: Se presentan los resultados de los grupos focales de alto y bajo rendimiento por categorías. Entre comillas, citas textuales que ejemplifican el discurso para cada categoría.

Fuente: Datos de la investigación 
El alumnado de alto rendimiento reporta una variedad de recursos o herramientas utilizados para apoyar la planificación diaria o semanal de tareas y contribuir al logro de los objetivos. En el caso de estudiantes con bajo rendimiento, también se emplean herramientas, aunque se hace referencia a una menor variedad de ellas. Las diferencias más claras se aprecian no en la frecuencia de uso, sino en la finalidad con que son empleadas. Entre los primeros, las herramientas utilizadas para la gestión de su tiempo constituyen apoyos externos que contribuirían a la regulación de las tareas, el cumplimento de los plazos y sirven como elementos para el seguimiento y autoevaluación de la planificación temporal de actividades académicas. En cambio, los estudiantes de bajo rendimiento académico utilizan las herramientas de manera más limitada. Para ellos, preferentemente cumplen la función de actuar como recordatorios inmediatos, no como un mecanismo para organizar y controlar el tiempo y los objetivos fijados.

Tanto el alumnado de bajo como alto rendimiento valora la importancia de la gestión del tiempo para el éxito en sus estudios. Sin embargo, sus percepciones acerca de la eficacia con que gestionan el tiempo difieren. Los estudiantes con alto rendimiento consideran que pueden gestionar el tiempo de forma adecuada, obteniendo beneficios en términos de aprendizaje y evitando la presión derivada de contar con un cúmulo de tareas pendientes no abarcables. El alumnado de bajo rendimiento no muestra niveles de autoconfianza similares, asumiendo su mala gestión del tiempo, que justifican en parte por la imposibilidad de organizar adecuadamente un conjunto de actividades que desbordan los límites del tiempo disponible.

\section{Discusión y conclusiones}

Este trabajo ha explorado la relación existente entre gestión del tiempo y rendimiento académico en el alumnado universitario, obteniendo una correlación significativa entre ambas variables. Confirmaríamos por tanto los resultados obtenidos en estudios anteriores (BASILA, 2014; DURÁN-APONTE; PUJOL, 2012; GARCÍA-ROS; PÉREZ-GONZÁLEZ, 2012; MACCANN; FOGARTY; ROBERTS, 2012). Sin embargo, la intensidad de esta relación $(r=0,122)$ no es elevada y se sitúa en niveles próximos a los hallados en la mayoría de los trabajos precedentes, donde la asociación entre ambas variables alcanza valores discretos. Durán-Aponte y Pujol (2012) hallaron correlaciones comprendidas en el rango de 0,077 a 0,124 entre las sub-escalas del TMBQ y el rendimiento académico al término del primer trimestre de estudios universitarios. Barrera, Donolo y Rinaudo (2008) obtuvieron correlaciones que no superaron el valor 0,210, revelando la existencia de cierta tendencia a que el alumnado con mejor rendimiento gestione mejor su tiempo. En la revisión de trabajos sobre la gestión del tiempo, realizada por Claessens y otros autores (2007), se llegó a concluir incluso que la relación con el rendimiento académico no es clara. Una excepción a los trabajos citados es el que llevaron a cabo García-Ros y PérezGonzález (2012), sobre una muestra de estudiantes universitarios de nuevo acceso a los que aplicaron una adaptación española del TMBQ. Tres de las sub-escalas de este instrumento correlacionaron significativamente con el rendimiento académico medio al término del primer año de estudios, alcanzándose valores de hasta $\mathrm{r}=0,40$.

Según Barrera, Donolo y Rinaudo (2008) la baja correlación que ha venido siendo habitual, y que aquí hemos constatado, puede tener una posible explicación en el hecho 
de que el alumnado universitario es bastante homogéneo. A pesar de las diferencias en las calificaciones, haber accedido a la universidad implica una trayectoria académica previa, unas habilidades y motivaciones que no poseen quienes no lo intentaron o no lo lograron. Es respecto a este tipo de sujetos donde podrían aparecer diferencias más claras y significativas en la gestión del tiempo. De cara a contrastar esta hipótesis, sería interesante estudiar la gestión del tiempo entre alumnado de enseñanza secundaria, diferenciando en función de su éxito o fracaso académico. Por otra parte, debe tenerse en cuenta que la variable rendimiento se asocia a un conjunto amplio de variables personales, familiares, académicas y contextuales. En consecuencia, raramente una sola variable presenta correlaciones elevadas con los resultados de aprendizaje, y la gestión del tiempo no constituye una excepción.

El empleo de la TMBQ para medir la gestión del tiempo nos permite atender a la multidimensionalidad de este constructo, considerando el comportamiento de las diferentes sub-escalas presentes en el instrumento. En este sentido, al comparar las puntuaciones logradas por subgrupos de alumnado con bajo y alto nivel de rendimiento, las diferencias no son importantes. La sub-escala percepción sobre el control del tiempo es la única donde las diferencias observadas son estadísticamente significativas. Precisamente, esta sub-escala es la que mayor correlación con el rendimiento académico arrojó en el estudio inicial de los autores del TMBQ (MACAN et al., 1990), con un valor de 0,22. En relación al resto de las sub-escalas, solo puede afirmarse una ligera tendencia a que los estudiantes de alto rendimiento obtengan mejores puntuaciones en herramientas para la gestión del tiempo. Esta sub-escala alcanzó la correlación más alta con rendimiento académico $(\mathrm{r}=0,124 ; \mathrm{p}<0,05)$ en el estudio de Durán-Aponte y Pujol (2012), si bien mientras que en el presente estudio ha participado alumnado de nuevo ingreso, el trabajo citado se centró sobre alumnado universitario de los últimos cursos de su plan de estudios.

El empleo de grupos focales nos ha permitido complementar los resultados cuantitativos con las perspectivas de los estudiantes de bajo y alto nivel de rendimiento. La triangulación metodológica permite reforzar la validez de resultados tales como la existencia de una cierta relación entre el rendimiento y la percepción sobre un eficaz control del tiempo. Además, la riqueza de significados aportados por el análisis de los grupos focales permite detectar aspectos concretos en función de los cuales uno y otro tipo de alumnado difieren al manejar su tiempo, y que no son captados por el TMBQ. Por ejemplo, aunque en la sub-escala establecimiento de objetivos y prioridades no se observaban diferencias significativas, los discursos de grupo revelan distintos comportamientos en lo que respecta al cumplimiento de la planificación establecida. Entre el alumnado de bajo rendimiento, la planificación de tareas resulta poco efectiva. Las razones se encontrarían en un cálculo erróneo sobre el tiempo que les llevará la realización de ciertas actividades, las interrupciones, la carencia de automonitoreo respecto a un objetivo preciso, o la falta de adaptación a acontecimientos inesperados.

También caracteriza al alumnado de alto rendimiento su mayor grado de estructuración del tiempo, separando las tareas de estudio del resto de actividades y ubicándolas en espacios concretos de sus rutinas horarias semanales. El desarrollo de este tipo de habilidades no constituye sólo un beneficio para la gestión efectiva de las horas de estudio, sino también para una mejor gestión de las relaciones sociales y actividades de ocio. El efecto logrado redunda en un control integral de la vida, favoreciendo el bienestar psicológico y la salud (KAYA et al., 2012). 
Las diferencias en el manejo de herramientas para la gestión del tiempo no son significativas en lo que respecta a la frecuencia de su utilización, medida por el TMBQ. Sí lo son, en cambio, en la función a la que responde su uso, la cual queda evidenciada en los discursos de grupo. Los resultados obtenidos nos llevan a afirmar que las herramientas para la gestión del tiempo se utilizan con mayor claridad al servicio de la autorregulación del aprendizaje en el caso de los estudiantes de alto rendimiento.

Ya hemos señalado que, en la línea de algunas evidencias aportadas por trabajos anteriores (CLAESSENS et al., 2007), las correlaciones encontradas entre las dos variables en estudio muestran poca intensidad. De acuerdo con este resultado, queda algo limitada la posibilidad de lograr una adecuada explicación y un buen pronóstico del rendimiento a partir de las habilidades de gestión del tiempo (BARRERA; DONOLO; RINAUDO, 2008). Sin embargo, considerando también los resultados cualitativos obtenidos en este trabajo, apoyamos la vinculación que parte de la literatura sobre este tema ha establecido entre las dos variables estudiadas. Con base en esa vinculación, se ha llegado a sugerir que los comités para la selección de estudiantes universitarios deberían evaluar la habilidad en la gestión del tiempo como parte de las condiciones de entrada (GEORGE et al., 2008). La autorregulación de la gestión del tiempo constituiría, por tanto, una variable presagio del éxito académico. Ha resultado ser un factor determinante para la adaptación del estudiante a la universidad (SOARES; ALMEIDA; GUISANDEZ, 2011) y para la reducción del estrés que pueden generar las exigencias que le plantea el sistema educativo universitario (HÄFNER et al., 2014).

De acuerdo con ello, consideramos que se deben ofertar a los estudiantes de primer año universitario talleres o cursos dirigidos a la mejora de sus habilidades de gestión del tiempo. Este tipo de actividades contribuiría a su adaptación al contexto universitario y podría tener un impacto positivo sobre su éxito académico. En particular, es necesario entrenar al alumnado en el uso de una variedad de herramientas para la planificación del tiempo académico y para su seguimiento. Este ámbito ha resultado ser el más deficitario en el conjunto de estudiantes, con un menor desarrollado entre el alumnado de bajo rendimiento. La intervención dirigida a mejorar estas habilidades habría de contemplar un entrenamiento sistemático del alumnado en el análisis de las tareas académicas que debe afrontar, valorando las limitaciones temporales para su realización y los recursos personales o materiales disponibles para ello. El alumnado debe desarrollar su capacidad para, a partir de este análisis, establecer objetivos a corto plazo dividiendo las tareas más complejas en subtareas simples, fijando fechas límite y estableciendo de manera realista un plan o secuencia temporalizada de actuaciones que le lleve a cumplir con los requerimientos académicos. Es fundamental el seguimiento de la ejecución del plan de trabajo, a través de una revisión periódica de lo realizado, que permita constatar logros, atender a contingencias imprevistas, analizar las causas de posibles retrasos o incumplimientos, y reformular actuaciones. Es precisamente el incumplimiento de la planificación de tareas uno de los rasgos que caracterizan al alumnado de bajo rendimiento, de acuerdo con los resultados obtenidos en el presente estudio. Herramientas como la lista de control de tareas, la agenda o el diario resultarán de utilidad para la planificación del tiempo y su seguimiento, propiciando la necesaria reflexión crítica para fortalecer la conciencia del estudiante sobre su trabajo diario y su autoconfianza en la gestión temporal del mismo. 
De cara a futuros estudios, sería interesante valorar el papel de las variables implicadas en la eficacia de las estrategias formativas. Puesto que no todos los individuos se ven beneficiados igualmente por un programa de entrenamiento en habilidades para la gestión del tiempo (MACAN et al., 2010), optimizar los beneficios de este tipo de programas requerirá un análisis de las variables personales que moderan la relación entre gestión del tiempo y resultados académicos.

\section{Referencias}

BARRERA, María Laura; DONOLO, Danilo Silvio; RINAUDO, María Cristina. Ritmo de estudio y trayectoria universitaria. Anales de Psicología, Murcia, v. 24, n. 1, p. 9-15, 2008. Disponible en: <http://revistas.um.es/ analesps/article/view/31651>. Acceso: 3 nov. 2015.

BASILA, Catherine. Good time management and motivation level predict student academic success in college on-line courses. International Journal of Cyber Behavior, Psychology \& Learning, v. 4, n. 3, p. 45, 2014.

BRITTON, Bruce; TESSER, Abraham. Effects of time-management practices on college grades. Journal of Educational Psychology, n. 83, n. 3, p. 405-410, 1991.

CLAESSENS, Brigitte et al. A review of the time management literature. Personnel Review, United Kingdom, v. 36, n. 2, p. 255-276, 2007.

COHEN, Jacob. Statistical power analysis for the behavioral sciences. Hillsdale: Lawrence Erlbaum Associates, 1988.

DUNLOSKY, John; HERTZOG, Cristopher. Updating knowledge about encoding strategies: a componential analysis of learning about strategy effectiveness from task experience. Psychology and Aging, v. 15, n. 3, p. 462-474, 2000.

DURÁN-APONTE, Emylse; PUJOL, Lydia. Estilos de aprendizaje, gestión del tiempo y rendimiento académico en estudiantes universitarios. In: CONGRESO MUNDIAL DE ESTILOS DE APRENDIZAJE, 5., 2012, Santander. Anales.... Santander: Universidad de Cantabria, 2012. Disponible en: <http://dialnet.unirioja.es/servlet/ articulo?codigo=4635078>. Acceso: 3 nov. 2015.

DURÁN-APONTE, Emylse; PUJOL, Lydia. Manejo del tiempo académico en jóvenes que inician estudios en la Universidad Simón Bolívar. Revista Latinoamericana de Ciencias Sociales, Niñez y Juventud, v. 11, n. 1, p. 93-108, 2013.

EILAM, Billie; AHARON, Irit. Students planning in the process of self-regulated learning. Contemporary Educational Psychology, v. 28, p. 304-34, 2003.

FORBUS, Patricia; NEWBOLD, John; MEHTA, Sanjay. A study of non traditional and traditional students in terms of their time management behaviors, stress factors, and coping strategies. Academy of Educational Leadership Journal, v. 15, n. 2, p. 67-71, 2011. Disponible en: <http://www.alliedacademies.org/public/ journals/ journaldetails. aspx?jid=5>. Acceso: 3 nov. 2015. 
GARCÍA-ROS, Rafael; PÉREZ-GONZÁLEZ, Francisco. Spanish version of the time management behavior questionnaire for university students. The Spanish Journal of Psychology, Madrid, v. 15, n. 3, p. 1485-1494, 2012.

GEORGE, Darren et al. Time diary and questionnaire assessment of factors associated with academic and personal success among university undergraduates. Journal of American College Health, v. 56, p. 706-715, 2008.

HÄFNER, Alexander et al. Stress prevention through a time management training intervention: an experimental study. Educational Psychology, Hong Kong, v. 34, n. 3, p. 403-416, 2014.

HAMDAN, Azura et al. Time management does not matter for academic achievement unless you can cope. International Proceedings of Economics Development and Research, Taiwan, v. 78, n. 22, p. 22-26, 2014.

KAYA, Hatice et al. Assessing time-management skills in terms of age, gerder, and anxiety levels: a study on nursing and midwifwry students in Turkey. Nurse Education in Practice, United Kingdom, v. 12, p. 284-288, 2012.

KITSANTAS, Anastasia; WINSLER, Adam; HUIE, Faye. Self-regulation and ability predictors of academic success during college: a predictive validity study. Journal of Advanced Academics, v. 20, n. 1, p. 42-68, 2008.

KRUEGER, Richard; CASEY, Mary Anne. Focus groups: a practical guide for applied research. Newbury Park: Sage, 2014.

LIU, Ou Lydia et al. The assessment of time management in middle-school students. Personality and Individual Differences, United Kingdom, v. 47, n. 3, p. 174-179, 2009.

MACAN, Therese et al. College students' time management: correlations with academic performance and stress. Journal of Educational Psychology, v. 82, n. 4, p. 760-768, 1990.

MACAN, Therese; GOBSON, Janet; CUNNINGHAM, Jennifer. Will you remember to read this article later when you have time? The relationship between prospective memory and time management. Personality and Individual Differences, Kingdom, v. 48, n. 6, p. 725-730, 2010.

MACCANN, Carolyn; FOGARTY, Gerard; ROBERTS, Richard. Strategies for success in education: time management is more important for part-time than full-time community college students. Learning and Individual Differences, v. 22, n. 5, p. 618-623, 2012.

METCALFE, Janet; KORNELL, Nate. A region of proximal learning model of study time allocation. Journal of Memory and Language, v. 52, n. 4, p. 463-477, 2005.

OLOWOOKERE, Elizabeth et al. Time management practices, character development and academic performance among University Undergraduates: covenant university experience. Creative Education, v. 6, n. 1, p. 79-86, 2015.

PEHLIVAN, Abdülkadir. The effect of the time management skills of students taking financial accounting course on their course grades and grade point averages. International Journal of Business and Social Science, v. 4, n. 5, p. 196-199, 2013. Disponible en: <http://ijbssnet.com/index.php/archive>. Acceso: 4 nov. 2015. 
PINTRICH, Paul et al. Reliability and predictive validity of the Motivated Strategies for Learning Questionnaire (MSLQ). Educational and Psychological Measurement, v. 53, n. 3, p. 801-813. 1993.

ROBBINS, Steve et al. Intervention effects on college performance and retention as mediated by motivational, emotional, and social control factors: integrated meta-analytic path analyses. Journal of Applied Psychology, v. 94, n. 5, p. 1163-1184, 2009.

ROBOTHAM, David. Student part-time employment: characteristics and consequences. Education + Training, United Kingdom, v. 54, n. 1, p. 65-75, 2012.

SOARES, Ana Paula; ALMEIDA, Leandro; GUISANDEZ, Adelina. Ambiente académico y adaptación a la universidad: un estudio con estudiantes de $1^{\circ}$ año de la Universidad Do Minho. Revista Iberoamericana de Psicología y Salud, La Coruña, v. 2, n. 1, p. 99-121, 2011. Disponible en: <http://suips.webs.uvigo.es>. Acceso: 3 nov. 2015.

SON, Lisa; KORNELL, Nate. Simultaneous decisions at study: time allocation, ordering, and spacing. Metacognition and Learning, v. 4, n. 3, p. 237-248, 2009.

UKPONG, Eno; GEORGE, Ime. Length of study-time behavior and academic achievement of social studies education students in the University of Uyo. International Education Studies, v. 6, n. 3, p. 172-178, 2013.

WILHITE, Stephen. Self-efficacy, locus of control, self-assessment of memory ability, and study activities as predictors of college course achievement. Journal of Educational Psychology, v. 82, n. 4, p. 696-700, 1990.

ZIMMERMAN, Barry. Enhancing student academic and health functioning: a self-regulatory perspective. School Psychology Quarterly, v. 11, n. 1, p. 47-66, 1996.

Recibido en: 18.12.2015 Aprobado en: 11.10.2016

Angélica Garzón Umerenkova es doctora en ciencias de la educación por la Universidad de Sevilla (España). Profesora en la Universidad El Bosque, de Bogotá (Colombia).

Javier Gil Flores es doctor en ciencias de la educación por la Universidad de Sevilla (España). Profesor en la Universidad de Sevilla (España). 